\title{
Nachhaltige Landwirtschaft und die BSE-Krise
}

\author{
Die deutsche und europäische Agrarpolitik \\ folgen weltweiten Tendenzen der Liberalisie- \\ rung und der Umweltintegration. Wider- \\ sprüchliche Anforderungen und Diskurse ver- \\ unsichern Landwirte wie Verbraucher. Eine \\ prozessorientierte, reflexive Agrarpolitik könn- \\ te Abhilfe schaffen. \\ Von Peter H. Feindt
}

$D^{i}$ e Landwirtschaft der hoch industrialisierten Länder befindet sich seit Jahrzehnten in einem Prozess der Rationalisierung, Spezialisierung und Konzentration. Der technischökonomische Modernisierungsprozess ging einher mit einschneidenden Veränderungen der ländlichen Räume, der Arbeitsprozesse, des Alltagslebens, der Ökosysteme und der ökonomischen Situation der Landwirte. Viele dieser Entwicklungen werden von den Betroffenen oder von gesellschaftlichen Gruppen als ambivalent oder krisenhaft wahrgenommen.

Die BSE-Krise der Jahreswende 2000/2001 wurde von der rot-grünen Bundesregierung mit einer radikalen Neudefinition der Agrarpolitik unter dem Primat des Verbraucherschutzes beantwortet. Diese „Agrarwende“ setzte programmatisch vor allem auf eine Ausweitung des ökologischen Landbaus sowie die Stärkung regionaler Kreisläufe und des ländlichen Raums, strategisch auf eine Kooperation der verschiedenen Akteure und eine veränderte Verbrauchernachfrage („Magisches Sechseck“). Kritiker warfen diesem Politikansatz mangelndes Verständnis landwirtschaftlicher Produktionsprozesse, eine Vernachlässigung ökonomischer und politischer Rahmenbedingungen sowie übermäßige Orientierung an populären, aber unzutreffenden Vorstellungen von Landwirtschaft vor. Beide Seiten beanspruchen, nachhaltige Entwicklungspfade für eine moderne Landwirtschaft zu entwickeln.

\section{Mediendiskurs und Krise}

Die Ausgangsthese des Projekts „AgChange“ war, dass anhand der BSE-Krise und der „Agrarwende“ divergierende Deutungen der krisenhaften Phänomene im Agrarbereich sowie unterschiedliche Bewertungen landwirtschaftlicher Entwicklungsoptionen zum Ausdruck kommen (1). Zudem sind Agrarreformen immer mit Verteilungskonflikten verbunden. Die Frage des Stellenwerts von Naturschutz, ökologischer, konventioneller und gentechni- scher Landwirtschaft ist außerdem mit Konflikten über die Nutzung der Flächen verbunden. Im Sinne eines transdisziplinären Forschungsansatzes wurden mehr als zweihundert Praxispartner aus Landwirtschaft, Verbraucherschutz, Politik und Verwaltung frühzeitig und dialogisch einbezogen.

Die „Agrarwende“ wurde von der Bundesregierung unter Berufung auf eine veränderte öffentliche Wahrnehmung legitimiert. Im Projekt „AgChange“ haben wir unter Leitung von Daniela Kleinschmit und Peter H. Feindt eine Vollerhebung der Berichterstattung über BSE und Agrarpolitik in den fünf überregionalen deutschen Tageszeitungen für die Jahre 2000 bis 2005 vorgenommen. Dabei haben wir insgesamt mehr als 7.000 Artikel ausgewertet. Im Ergebnis zeigte sich, dass in den Jahren 2000 und 2001 das Thema BSE zwar den Agrardiskurs dominierte. In den Medien prägten aber nicht sorgende Bürger und Nichtregierungsorganisationen den Diskurs, sondern mächtige Akteure aus dem Zentrum des politischen Systems. Vor allem die Bundes- und Landesregierungen und die EUKommission nutzten ihre Deutungsmacht, um sich als Problemlöser zu präsentieren. Sie wurden jedoch zugleich am häufigsten als Verursacher der Probleme benannt. Als Opfer erscheinen im medialen BSE- und Agrar-Diskurs vor allem die Verbraucher und die Gesellschaft. Dies erklärt die starke Mobilisierungsfähigkeit des Themas.

Eine Interpretation der Daten vor dem Hintergrund der These einer Mediatisierung der Politik zeigt, dass Lebensmittelkrisen wie BSE geeignet sind, eine umfassende Berichterstattung über die Agrarpolitik auszulösen, die sich aufgrund ihres technischen Gehalts und ihrer Komplexität ansonsten eher der medialen Aufmerksamkeit entzieht. In Krisensituationen erzeugt die mediale Berichterstattung kurzfristige Handlungserwartungen an die Politik. Während der BSE-Krise kam die etablierte Agrarpolitik dem nur unzureichend nach. Kanzler Schröder nutzte diese Schwäche zu einer personellen und organisatorischen Reform, die im Routine-Modus der Politik undenkbar gewesen wäre. Für den Zusammenhang von Demokratie und Öffentlichkeit positiv zu werten ist der Umstand, dass eine aus Sicht der Verbraucher bedrückende Problemlage in den Medien erfolgreich zu einem politischen Thema verdichtet wurde. Die Ängste der Verbraucher waren in der BSE-Krise jedoch ihrerseits Ergebnis medialer Vermittlung (Feindt/Kleinschmit 2007).

\section{Weltweiter Paradigmenwechsel}

Die „Agrarwende“ stellt kein singuläres politisches Ereignis dar, sondern ist Teil einer Veränderung der Agrarpolitik, die $\rightarrow$ 


\section{„Die derzeitige Agrarpolitik stellt einen Mittelweg zwischen Aufrechterhaltung der Landnutzung, Einkommensstützung und nur mäßigen Anreizen zur Optimierung der ökologischen Leistungen dar."}

sich seit etwa Mitte der 1980er Jahre vollzieht. In allen Ländern der Organisation für wirtschaftlichen Zusammenarbeit und Entwicklung (OECD) gerät das seit den 1930er Jahren dominante Paradigma einer Landwirtschaft, die aufgrund struktureller und naturbedingter Nachteile von Staatshilfe abhängig ist, angesichts von Produktionsüberschüssen, Umweltschäden und hoher öffentlicher Kosten in die Defensive. Seitdem ist ein Trend fort von einer protektionistischen und produktivistischen Agrarpolitik zu beobachten. Man kann daher von Politik-Konvergenz sprechen - die Agrarpolitiken werden ähnlicher. Neben ähnlichem Problemdruck spielen dabei Harmonisierungsprozesse durch die Integration des Agrarhandels in das Allgemeine Zollund Handelsankommen (GATT) und die Welthandelsorganisation (WTO), die EU-Osterweiterung sowie transnationale Kommunikation in der OECD eine wichtige Rolle. Diese Mechanismen führen $\mathrm{zu}$ einer Senkung und Angleichung des Stützungsniveaus für landwirtschaftliche Erzeuger und zu einer Umstrukturierung der Agrarpolitik hin zu weniger produktionsbezogenen Maßnahmen.

Im internationalen Vergleich gibt es aber deutliche Unterschiede. Während in den USA, Neuseeland und Australien der Marktliberalismus an Einfluss gewinnt, setzt die EU auf eine multifunktionale Landwirtschaft, deren öffentliche Leistungen, etwa für das Landschaftsbild, eine staatliche Unterstützung rechtfertigen. Seit 1995 gewinnt weltweit zudem das Paradigma einer globalen Landwirtschaft an Einfluss, das vom Verbraucher her denkt und Landwirte als Teil potenziell globaler Produktionsnetzwerke sieht. Politik hat hier die Sicherung von Standards und Qualität zum Ziel und setzt dazu auf Harmonisierung, Wettbewerbspolitik sowie den Schutz von intellektuellem Eigentum und von Investitionen.

Während die „Agrarwende“ Ideen des Multifunktionalismus und des Globalismus aufnimmt, enthält die Reform der Gemeinsamen Agrarpolitik (GAP) der EU von 2003 Elemente aller vier Paradigmen: Die Entkopplung der Direktzahlungen von der Produktion dient der Liberalisierung, ihre Verknüpfung mit Umwelt- und anderen Vorschriften (Cross Compliance) der Multifunktionalität. Dass die Landwirte für die Einhaltung gesetzlicher Vorschriften Geld erhalten, zeigt das Fortleben der Idee einer staatsabhängigen Landwirtschaft. Die zunehmende Einführung von Mindeststandards in den Bereichen Tier-, Umwelt-, Natur- und Verbraucherschutz und von speziellen Standards für Öko-Produkte entspricht dem Globalitätsparadigma. Gleiches gilt für die Kennzeichnung von Produkten regionaler Herkunft, von traditionellen Lebensmitteln sowie von Produkten mit und ohne Gentechnik. Auch die obligatorischen Rückverfolgbarkeitssysteme passen ins Globalitätsparadigma zumal sich die EU bemüht, diese Systeme und Regeln in der WTO abzusichern beziehungsweise als globalen Standard durchzusetzen. Nach der EU-Osterweiterung ist daher auch keine Wiederkehr der Kleinbauern in der Agrarpolitik zu erwarten. Wie Franziska Müllers Analysen zeigen, wird die Semi-Subsistenzwirtschaft in Teilen Osteuropas nur dann von der Agrarpolitik profitieren, wenn sie multifunktionale Leistungen erbringt oder sich an lokalen Märkten orientiert.

Im Ergebnis ist in der europäischen Agrarpolitik jedoch eine zunehmende Integration von Umweltbelangen zu konstatieren. Die Integration umweltpolitischer Ansätze und Instrumente in die GAP half, andere Probleme der Agrarpolitik wie Budget, Handelskonflikte und die Legitimation der Einkommenstransfers zu lösen. Der Handlungsspielraum für Reformen der GAP ist jedoch dadurch beschränkt, dass Umverteilungswirkungen zwischen den Mitgliedstaaten vermieden werden müssen, um die notwendigen qualifizierten Mehrheiten im Agrarrat zu erreichen (Feindt 2007).

\section{Policy-Szenarien}

Im Rahmen einer modellgestützten Analyse hat Rainer Sodtke die Auswirkungen verschiedener Szenarien der Agrarförderpolitik auf Landnutzung, Umweltqualität und Einkommenssituation der Betriebe in einer Modellregion in Brandenburg untersucht. Seine Wirkungsanalyse zeigt, dass die verschiedenen möglichen Ausgestaltungen der GAP-Reform 2003 nur geringe Unterschiede erzeugen. Die Produktionsintensitäten in Ackerbau und Tierhaltung nehmen ab, die betrieblichen Einkommen sinken leicht, die Umweltwirkungen verbessern sich jeweils leicht gegenüber 2004. Die Einstellung von Transferzahlungen bei einer vollständigen Liberalisierung hätte auf den ertragsschwachen Standorten der Modellregion hingegen extreme Einkommenseinbußen, großflächige Betriebsaufgaben und Flächenstilllegungen zur Folge. Die wenigen verbleibenden Betriebe würden sich mit intensiver Marktfruchtproduktion halten; bei fehlender Pflege der Stilllegungsflächen verschlechtern sich die Habitatqualitäten vieler Tier- und Pflanzenarten.

Eine vollständige Nutzung der Direktzahlungsmittel für Agrarumweltmaßnahmen hätte demgegenüber eine großflächig extensive Landwirtschaft mit einem sehr hohen Anteil an ökologischem Landbau und extensiver Grünlandnutzung zur Folge. Die betrieblichen Einkommen wären ähnlich hoch wie 2004 und aufgrund einer deutlich verminderten Intensität im Nährstoff- beziehungsweise Pflanzenschutzmitteleinsatz und der vielseitigeren Fruchtfolgen werden die Wirkungen auf fast 
alle Umweltqualitätsziele am günstigsten bewertet. Die Szenarien zeigen, dass eine konsequente Umsetzung der verschiedenen agrarpolitischen Paradigmen sehr unterschiedliche Auswirkungen auf die Landnutzung, Einkommen und Umweltqualität hätte. Die derzeitige Agrarpolitik stellt im Ensemble dieser Szenarien einen Mittelweg dar - Aufrechterhaltung der Landnutzung, Einkommensstützung, aber nur mäßige Anreize zur Optimierung der ökologischen Leistungen.

\section{Widersprüchliche Embleme}

Eine qualitative Analyse des agrarpolitischen Diskurses in den Jahren nach der "Agrarwende“ anhand von Interviews mit agrarpolitischen Akteuren 2003 und 2005 hat Sabine Weiland vorgenommen. Sie konzentriert sich dabei auf Embleme - also Begriffe und Bilder, die eine wörtliche mit einer interpretierten Bedeutung verknüpfen und dadurch einen Bedeutungssprung erzeugen. Bestimmte Embleme - wie „die Künast“ oder „Agrarwende“ - werden von allen Akteuren verwendet. Sie prägen den Diskurs in hohem Maße. Die Sinnzuschreibungen fallen jedoch je nach Akteur und Perspektive sehr unterschiedlich aus. Die Vielfalt und Gegensätzlichkeit der agrarpolitischen Perspektiven reproduziert sich hier also auf der Ebene des Sprachgebrauchs. Agrarpolitische Akteure verwenden die gleichen Begriffe, verbinden damit aber zum Teil sehr unterschiedliche Vorstellungen und Wertungen.

Auch „Natur" und „Arbeit" erscheinen aus lebensweltlicher Perspektive vielfältig. Dies ergab Tanja Mölders' Auswertung von Tiefeninterviews mit 22 Landwirt(inn)en, die im Winter 2003/2004 im Großraum Hamburg geführt wurden. „Natur“ stellt beispielsweise die Gesamtheit der natürlichen Produktionsfaktoren wie auch Heimat dar. „Arbeit“ ist neben Erwerbsarbeit auch Versorgungsarbeit, Eigenarbeit und bürgerschaftliches Engagement. Eine reproduktionstheoretische Interpretation der Ergebnisse macht deutlich, dass diese vielfältigen, lebensweltlichen Verständnisse und Konzepte von "Natur“ und „Arbeit" nicht gleichberechtigt nebeneinander stehen. Es findet eine Bewertung und Hierarchisierung statt, wonach solche Leistungen von „Natur" und "Arbeit", die nicht monetär in Wert gesetzt werden können, als reproduktiv abgespalten und abgewertet werden. Zugleich werden sie als Bedingungen einer funktionierenden landwirtschaftlichen Produktion vorausgesetzt.

\section{Verunsicherte Landwirte}

Auf Basis der Interviews bestätigt Tanja Mölders auch frühere Befunde einer starken Verunsicherung in den landwirtschaftlichen Familienbetrieben. Diese spitzt sich angesichts von BSEKrise, „Agrarwende“, GAP-Reform 2003 und der schlechten wirtschaftlichen Situation zum Zeitpunkt der Gespräche akut zu, was sich in dem Gefühl ausdrückt, dass es „eng wird“. Die Befragten sahen sich angesichts von Liberalisierung und neuen technischen Entwicklungen vor einer ungewissen ökonomischen Zukunft. Sie fühlten sich von der Agrarpolitik im Stich gelassen, von der Gesellschaft unverstanden und isoliert. Der Einfluss der unterschiedlichen agrarpolitischen Paradigmen wird von den Landwirt(inn)en als Addition widersprüchlicher Anforderungen wahrgenommen. In der Folge wirkten sie zum Teil orientierungslos: Wie sollen sie ihre internationale Wettbewerbsfähigkeit steigern und zugleich hohe Umweltstandards erfüllen?

Tatsächlich erhöhen die GAP-Reformen die Anforderungen an die landwirtschaftlichen Betriebsleiter und ihre Berater, eine stärkere Marktorientierung mit präziseren und zum Teil höheren Standards im Umwelt-, Verbraucher-, Tier-, und Naturschutz zu verbinden. Daher haben Rainer Sodtke und Manuel Gottschick im Winter 2005/2006 eine Untersuchung zu den Anforderungen an Agrarsoftware für landwirtschaftliche Beratung durchgeführt (Sodtke/Gottschick 2007).

Die Ergebnisse zeigen eine deutliche Diskrepanz zwischen den Bedürfnissen der Berater und den wissenschaftlichen und kommerziellen Angeboten zur Entscheidungsunterstützung. Dem Trend der Wissenschaft, immer aufwendigere und umfassendere Softwaretools zu erstellen, steht die Nachfrage nach einfachen und flexiblen Anwendungen gegenüber. Dabei geht es nicht um Probleme der Softwareergonomie oder mangelnder Schulung. Die Interviews mit 30 landwirtschaftlichen Beratern ergaben, dass vorrangig eigene Excel-Anwendungen verwendet werden. Zur Entscheidungsfindung wird jedoch keine entscheidungsunterstützende Software im engeren Sinn eingesetzt. Eine wichtige zukünftige Aufgabe besteht darin zu eruieren, wie der Bedarf der Berater an praktikabler Software mit einem Impuls für nachhaltigere Landwirtschaft verbunden werden kann.

\section{Partizipative Modellierung}

Agrarpolitik findet zunehmend in Wechselwirkung mit anderen Politikfeldern sowie in einem politischen Mehrebenensystem statt. Dies wird am Beispiel der Reform der europäischen Zuckermarktordnung vom Dezember 2005 deutlich, die in einem engen Zusammenhang mit der Entwicklungspolitik für die Länder Afrikas, der Karibik und des Pazifik (AKP) sowie für die am wenigsten entwickelten Länder (LDC) steht. Diese genießen derzeit im Rahmen begrenzter Quoten zollfreien Zugang zum ansonsten abgeschotteten europäischen Markt. Die europäische Zuckermarktordnung musste verändert werden, nachdem die EU im Oktober 2005 vor dem Streitschlichtungsausschuss der WTO endgültig einer Klage von Brasilien, Thailand und Australien mit Unterstützung weiterer Länder unterlegen war. Auch der Abschluss von Economic Partnership Agreements (EPA) der EU mit den AKP-Staaten steht unter Zeitdruck, weil 2008 eine Ausnahmeregel der WTO ausläuft.

Im Laufe des Jahres 2006 führte Manuel Gottschick eine Workshop-Reihe mit Zuckermarkt- und Entwicklungsexperten aus Bundesministerien und Nicht-Regierungsorganisationen sowie Wissenschaftlern durch - teilweise in Kooperation mit der Nord-Süd-Initiative von Germanwatch. Dabei zeigte sich, dass die Teilnehmer(innen) weniger an einer Verbesserung $\rightarrow$ 
der Auswirkungsanalysen der Zuckermarktreform interessiert waren als daran, die potenziellen Ergebnisse der laufenden EPAVerhandlungen abzuschätzen. Hierzu lagen jedoch keine belastbaren Studien vor. Außerdem erzeugte die Verschränkung verschiedener Politik- und Problemfelder weitere Unsicherheiten. Daher wurden Methoden der analytischen Modellierung und partizipative Verfahren unter Zuhilfenahme von analytischen Studien und weiteren Expert(inn)en kombiniert (Gottschick in review). Dies ermöglichte es den Teilnehmer(inn)en, die Problemstellung besser zu strukturieren und die Wechselwirkungen und Rückkopplungen zwischen den einzelnen Problembereichen zu berücksichtigen.

\section{Reflexive Agrarpolitik}

Mit der Agrarwirtschaft sind in den vergangenen Jahrzehnten auch die Interessenlagen, Akteurkonstellationen und ideellen Grundlagen in der Agrarpolitik vielfältiger geworden. Nach der BSE-Krise wurden daher in Politik und Medien sehr unterschiedliche Vorstellungen einer nachhaltigen Landwirtschaft artikuliert, die auf gegensätzlichen Naturkonzepten und gesellschaftlichen Ordnungsvorstellungen beruhen. Kaum thematisiert wurde, dass Nachhaltigkeit in verschiedenen Regionen und für verschiedene Arten von Betrieben sehr unterschiedliche Gestalt annehmen kann - dass nachhaltige Agrarpolitik sich also nicht an einem einzigen Modell orientieren kann, sondern die Vielfalt ermutigen und stimulieren sollte.

Um die differierenden Vorstellungen und Ansätze produktiv für Lernprozesse zu nutzen, wäre es notwendig, die Wahrnehmungen von Nebeneffekten in spezialisierten Sektoren und Organisationen zu verbessern. Dazu müssten beratende Foren geschaffen werden, in denen Landwirte, vor- und nachgelagerter Sektor mit Umweltschützern, Konsumenten und Vertretern des ländlichen Raums gemeinsam die Voraussetzungen und Implikationen von Praktiken thematisieren, gegebenenfalls problematisieren und verändern können. In solchen Foren, deren Teilnehmer die Vielfalt der betroffenen Lebensbereiche spiegeln, können beispielsweise auch Modellprojekte initiiert und begleitet oder die Ziele für innovative Forschungsprogramme formuliert werden.

Im Sinne einer solchen reflexiven Agrarpolitik war es sinnvoll, dass nach der BSE-Krise die Forschung zu alternativen Produktionssystemen und deren Förderung verstärkt wurde. Ähnliche Maßnahmen wurden aber beispielsweise auch in den Niederlanden ergriffen. Neuerungen stoßen dort zwar auch auf Probleme, wenn sie nicht in die Eigenlogik der Betriebe und Verbände passen, herkömmliche Wahrnehmungen herausfordern oder im Widerspruch zu machtvollen Interessen stehen. Sie stießen aber nicht auf eine dominant parteipolitische Aufladung.

Die Frage, ob eine andere rhetorische Präsentation der neuen Agrarpolitik mehr bewirkt hätte, ist schwer zu beantworten. Auf der Abschlusstagung unseres Projekts, die wir im März 2007 an der Evangelischen Akademie in Loccum unter Beteiligung von mehr als 50 Vertretern gesellschaftlicher Interessen und politisch-administrativer Amtsinhaber durchführten, wurde die Diagnose einer Pluralisierung der Agrarpolitik weitgehend geteilt (Feindt/Lange 2007). Das Konzept einer reflexiven Agrarpolitik wurde überwiegend wohlwollend aufgenommen. In den Diskussionen zeigte sich, dass die Vertreter ihre Positionen wechselseitig gut kennen und in ihrer Argumentation Ideen-Elemente aus unterschiedlichen agrarpolitischen Paradigmen verwenden. Der Dialog stieß jedoch dann an Grenzen, wenn zentrale Interessenlagen und (Um-)Verteilungsfragen berührt wurden. Zudem wurde deutlich, dass die Aufnahme von Argumenten anderer in das je eigene Problemverständnis durch die Eigenlogik der Diskurse von landwirtschaftlichen Interessenvertretern, Administratoren, Umweltverbänden und so weiter begrenzt war. Eine reflexive Agrarpolitik müsste hier daran arbeiten, Mechanismen zu stärken, die Folgenberücksichtigung im Sinne „reflexiver feedback-loops“ und das Re-Framing, die Re-Interpretation von Praktiken, erleichtern.

\section{Anmerkungen}

(1) Weitere Informationen auf der Projekthomepage: www.agchange.de. Wesentliche Ergebnisse und ausführlichere Darstellungen sind zu finden in: Feindt, P. / Gottschick, M. / Mölders, T. / Müller, F. / Sodtke, R. / Weiland, S. (Hrsg.): Nachhaltige Agrarpolitik als reflexive Agrarpolitik. (in Vorbereitung)

\section{Literatur}

Feindt, P.H.: Integration von Umweltbelangen in die europäische Agrarpolitik. Institutionelle Bedingungen und Politik-Lernen im Mehrebenensystem. In: Jacob, K. / Biermann, F. / Busch, P.-O. / Feindt, P.H. (Hrsg.): Politik und Umwelt. Wiesbaden 2007. i.E.

Feindt, P.H. / Kleinschmit, D.: Mediatisierung der Agrarpolitik? Die Rolle der Medien in der deutschen BSE-Krise. In: Koch-Baumgarten, S. / Mez, L. (Hrsg.): Medien und Politik - Neue Machtkonstellationen in ausgewählten Politikfeldern. Frankfurt 2007. S. 121-142.

Feindt, P.H. / Lange, J. (Hrsg.). Agrarpolitik im 21. Jahrhundert. Konflikte, Op tionen, Verständigungsbedarf. Loccum 2007.

Gottschick, M.: Participatory Sustainability Impact Assessment (pSIA): Scientific Policy Advice as a Social Learning Process. System Practice and Action Research (in review).

Sodtke, R.M. / Gottschick, M.: What Are the Needs to Agricultural Software? An Analysis of Experiences and Requirements of Agricultural Consultants. In: Parker, C. (Hrsg.): Proceedings of the EFITA/WCCA 2007 Joint Conference, 2-5 July 2007. Glasgow 2007.

\section{AUTOR + KONTAKT}

Dr. Peter H. Feindt arbeitet als Politikwissenschaftler am Forschungsschwerpunkt Biotechnik, Gesellschaft und Umwelt der Universität Hamburg. Bis Herbst 2007 leitet er dort die interdisziplinäre Nachwuchsgruppe "AgChange. Konflikte der Agrarwende“.

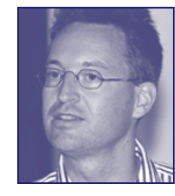

Universität Hamburg, Forschungsschwerpunkt Biotechnik, Gesellschaft und Umwelt, Ohnhorststraße 18, 22609 Hamburg. Tel.: +494042816613, E-Mail: phfeindt@botanik.uni-hamburg.de 
(c) 20I0 Authors; licensee IÖW and oekom verlag. This is an article distributed under the terms of the Creative Commons Attribution Non-Commercial No Derivates License (http://creativecommons.org/licenses/by-nc-nd/3.o/), which permits unrestricted use, distribution, and reproduction in any medium, provided the original work is properly cited. 